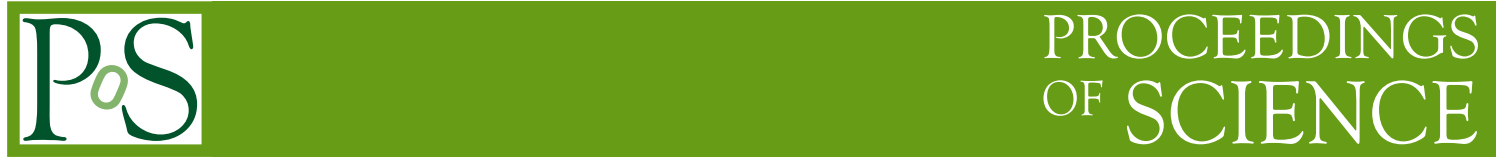

\title{
Precision measurement of charmed meson properties with the BABAR detector
}

\section{David R. Muller* for the BABAR collaboration}

SLAC National Accelerator Laboratory

E-mail: mullereslac.stanford.edu

\begin{abstract}
We present measurements of the mass of the $D^{0}$ meson, the natural line width of the $D^{*}(2010)^{+}$ meson, and the mass difference between the $D^{*}(2010)^{+}$and the $D^{0}$ mesons using pure samples of $D^{*}(2010)^{+} \rightarrow D^{0} \pi^{+}$decays. The measurements are based on a data sample corresponding to an integrated luminosity of about $477 \mathrm{fb}^{-1}$ collected with the BABAR detector. Large improvements with respect to the existing measurements have been obtained for all the measured quantities.
\end{abstract}

XXI International Workshop on Deep-Inelastic Scattering and Related Subject-DIS2013, 22-26 April 2013

Marseilles, France

\footnotetext{
* Speaker.
} 


\section{Introduction}

Masses and natural decay widths are among the most fundamental properties of bound states such as mesons. It is especially important to understand the lowest lying states of a particular flavor combination, which are sensitive to both the constituents and the binding mechanism. Here we present a new preliminary measurement of the mass of the $D^{0}$ meson, the ground state of the $c \bar{u}$ quark system, along with measurements [1] of the natural width of the $D^{*}(2010)^{+}$meson, the lowest vector state of the $c \bar{d}$ system, and the $D^{*+}-D^{0}$ mass difference. We use the decay chain $D^{*+} \rightarrow D^{0} \pi_{s}^{+}$, where $\pi_{s}^{+}$denotes a "slow" pion, and $D^{0} \rightarrow K^{-} K^{+} K^{-} \pi^{+}$for the $D^{0}$ mass measurement and $D^{0} \rightarrow K^{-} \pi^{+}$or $K^{-} \pi^{+} \pi^{-} \pi^{+}$for the $D^{*+}$ width and mass difference. Chargeconjugate decay chains are included, and implied throughout this article.

Improved measurements of such quantities require not only high statistics, but also an excellent understanding of the detector response. We focus on high signal:noise, while selecting events with a high resolution that can be constrained reliably from the data. Two approaches to this are to apply very stringent reconstruction and identification requirements to the tracks, and to select highmomentum candidates. At our center-of-mass $(\mathrm{CM})$ energy of $10.6 \mathrm{GeV}$, the $D$ mesons from $B$ meson decay are limited to low momenta where combinatoric backgrounds are large. We select only the much higher-momentum $D$ mesons from $c \bar{c}$ events. To minimize experimental resolution, we choose decay modes with a low $Q$ value, i.e., modes in which the sum of the daughter particles' masses is close to the parent mass. These modes have lower statistics, but better systematics.

\section{Charmed meson candidate selection}

These measurements use a sample of $477 \mathrm{fb}^{-1}$ recorded by the BABAR experiment [2] at CM energies near $10.6 \mathrm{GeV}$. This corresponds to roughly 650 million produced $e^{+} e^{-} \rightarrow c \bar{c}$ events containing about 400 million $D^{*+} \rightarrow D^{0} \pi_{s}^{+}$decays. For the $D^{0}$ mass measurement, we use the decay mode $D^{0} \rightarrow K^{-} K^{+} K^{-} \pi^{+}$, denoted $3 K \pi$, with a $Q$ value of $250 \mathrm{MeV} / c^{2}$. We consider each set of four charged tracks with zero net charge, in which the two negatively charged tracks are well identified as $K^{-}$, one positively charged track is identified as a $K^{+}$and the other as a $\pi^{+}$. Then we apply a " $D^{*}$ tag", requiring an additional identified $\pi^{+}$in the event, denoted $\pi_{s}^{+}$, such that the "correct" mass difference, $\Delta m=m_{3 K \pi \pi_{s}}-m_{3 K \pi}<150 \mathrm{MeV} / c^{2}$ but the "wrong" mass difference $\Delta m^{\prime}=m_{3 K \pi \pi_{s}}-m_{3 K \pi_{s}}>150 \mathrm{MeV} / c^{2}$.

To optimize the signal:noise and mass resolution, we require: the $3 K \pi \pi_{s}^{+}$system momentum to exceed $2.5 \mathrm{GeV} / c$ in the $e^{+} e^{-} \mathrm{CM}$ frame; the $\pi_{s}^{+}$momentum to exceed $150 \mathrm{MeV} / c$ in the laboratory frame; and all tracks to have a polar angle with respect to the $e^{-}$beam direction satisfying $\cos \theta<0.89$ in the laboratory frame, which excludes the very forward region of the detector. We then perform a kinematic fit to the $3 K \pi \pi_{s}^{+}$system that constrains all vertices geometrically and the $D^{*}$ vertex to the interaction region, and we retain candidates with $\chi^{2}<20$. If more than one candidate in an event satisfies all these criteria, we retain the one with the lowest $\chi^{2}$ value. The selected sample contains roughly 4350 signal events with a peak signal:noise of 150 .

For the $D^{*}(2010)^{+}$width and mass difference measurements, the $D^{*+} \rightarrow D^{0} \pi_{s}^{+}$decay mode provides the low $Q$ value of $145 \mathrm{MeV} / c^{2}$, and we use the $D^{0} \rightarrow K^{-} \pi^{+}$and $D^{0} \rightarrow K^{-} \pi^{+} \pi^{-} \pi^{+}$ modes, which have relatively high branching fractions and give the best signal:noise. We consider 
combinations of one well identified $K^{-}$and two or four identified $\pi^{ \pm}$, and apply a " $D^{0}$ tag", requiring $1860<m_{K \pi(\pi \pi)}<1870 \mathrm{MeV} / c^{2}$ and, for the $D^{0} \rightarrow K^{-} \pi^{+} \pi^{-} \pi^{+}$mode, both wrong mass differences $m_{K \pi \pi \pi \pi_{s}}-m_{K \pi \pi \pi_{s}}>166.5 \mathrm{MeV} / c^{2}$.

The $K^{-} \pi^{+}\left(\pi^{-} \pi^{+}\right) \pi_{s}^{+}$system must have a momentum between 3.6 and $4.3 \mathrm{GeV} / c$ in the $\mathrm{CM}$ frame, the $\pi_{s}^{+}$momentum must exceed $150 \mathrm{MeV} / c$ in the laboratory frome, and all tracks must have $\cos \theta<0.89$. We perform a similar kinematic fit to the $K^{-} \pi^{+}\left(\pi^{-} \pi^{+}\right) \pi_{s}^{+}$system and retain the candidate in each event with the highest $\chi^{2}$ confidence level if it exceeds 0.001 . The selected $D^{0} \rightarrow K^{-} \pi^{+}\left(K^{-} \pi^{+} \pi^{-} \pi^{+}\right)$sample contains roughly 140 (175) thousand signal events with a peak signal:noise of 2700 (1130).

\section{Analysis and fits}

All aspects of the detector performance that affect the mass scale or resolution must be understood, and are measured where possible in the data. Particularly important, especially for $\pi_{s}^{+}$and other soft particles, is the energy loss experienced by a particle as it traverses the detector material. This is calculated in the track reconstruction, and can be studied using $K_{S}^{0}$ decays. We select $K_{S}^{0} \rightarrow \pi^{+} \pi^{-}$candidates with a decay vertex inside the beampipe and consistent with the decay chain $D^{*+} \rightarrow D^{0} \pi_{s}^{+}, D^{0} \rightarrow K_{S}^{0} \pi^{+} \pi^{-}$; the decay pion tracks have an environment and momentum spectrum similar to those of our signal tracks. Fitting the $\pi^{+} \pi^{-}$invariant mass spectrum in bins of the lower of the two pion momenta, we obtain the measured $K_{S}^{0}$ masses shown in Fig. 3a. There is a deviation from the nominal $K_{S}^{0}$ mass [3] as the lower pion momentum decreases. We apply empirical correction factors of: 1.003 to the value of the magnetic field; and 1.018 and 1.059 to the energy losses returned by the Kalman filter in the beampipe and vertex tracker, respectively. With these corrections, all the data are consistent with the nominal $K_{S}^{0}$ mass, as shown by the solid points in Fig. 3a. A systematic uncertainty is estimated using correction factors that shift the data to the $\pm 1 \sigma$ edges of the $K_{S}^{0}$ mass band in Fig. 3a.

The choice of fitting function is vital, since the width and mass results depend critically on the signal and background shapes, respectively. High signal:noise is a key feature here, but we must study the functions carefully and extensively, testing them on both simulation and data in other decay modes. The signal function is the convolution of the true lineshape with a resolution function. Since the $D^{0}$ decays weakly, its lineshape is a delta function and we describe the $D^{0} \rightarrow 3 K \pi$ invariant mass resolution with a Voigtian function, $P(m)=\frac{1}{\left(m-m_{0}\right)^{2}-\gamma^{2} / 4} \otimes e^{-\left(m-m_{0}\right)^{2} / 2 \sigma^{2}}$, which is a convolution of Cauchy and Gaussian functions, where $m_{0}$ is the mass to be measured, and $\sigma$ and $\gamma$ are free parameters describing the shape. For the background, we choose a simple exponential function, $B(m)=A e^{C m}$, with $A$ and $C$ free parameters.

The measured $3 K \pi$ invariant mass distribution is shown in Fig. $3 \mathrm{~b}$, along with the fit result. The fit has good quality and returns a total signal of $4345 \pm 70 D^{0} \rightarrow 3 K \pi$ decays. The fitted resolution parameter values are consistent with those from the simulation. The fitted $D^{0}$ mass is insensitive to choice of signal function. The small sensitivity to the backround function is discussed below, along with the other systematic uncertainties.

For the $D^{*}(2010)^{+}$width and mass difference, we fit the distribution of the measured mass difference $\Delta m=m_{K \pi(\pi \pi) \pi_{s}}-m_{K \pi(\pi \pi)}$. The lineshape is a relativistic $P$-wave Breit-Wigner function, corrected for phase space. The resolution function is a sum of three Gaussian functions and a term 

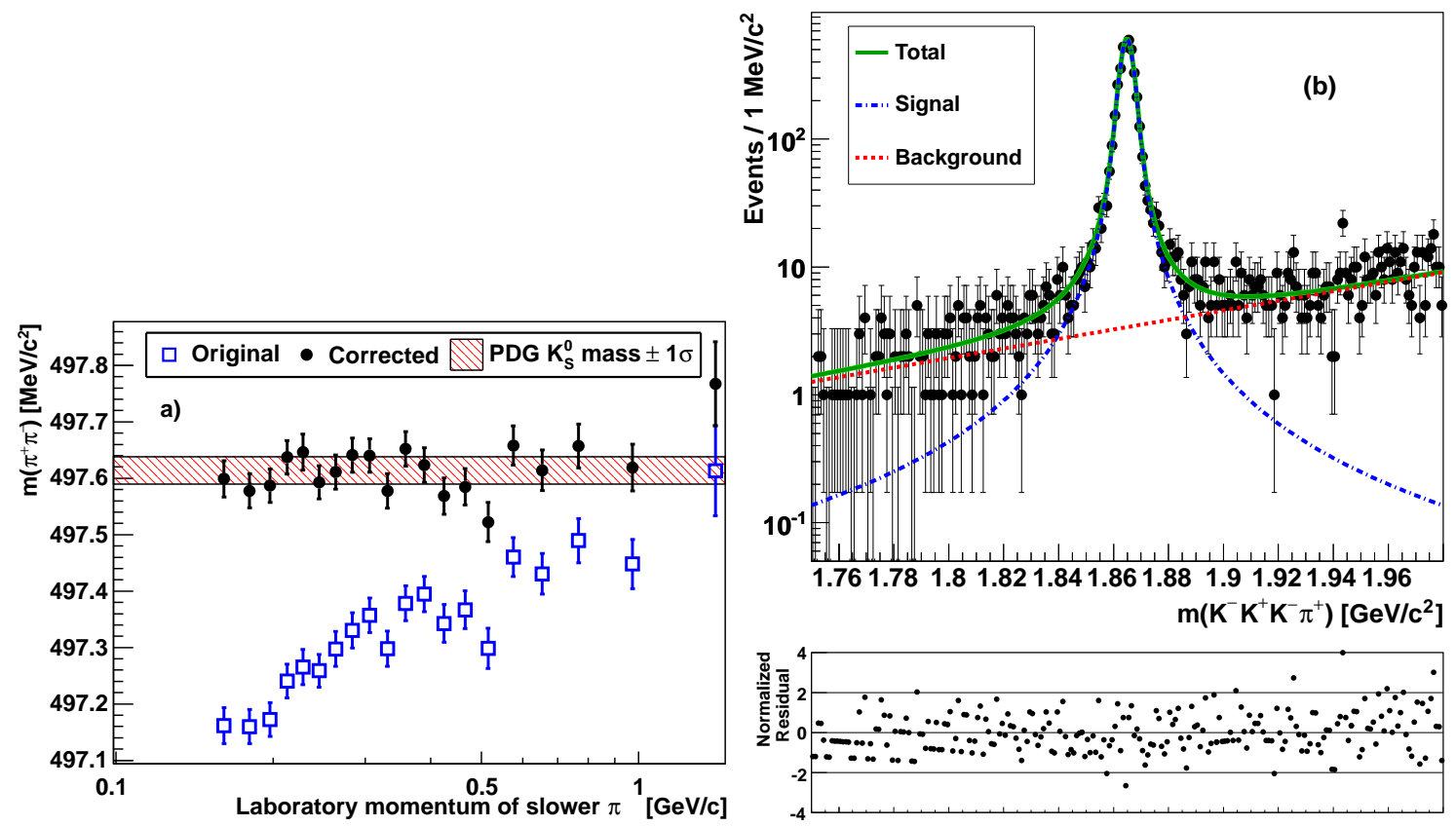

Figure 1: a) The average reconstructed $K_{S}^{0} \rightarrow \pi^{+} \pi^{-}$mass vs. the lower of the $\pi^{+}$and $\pi^{-}$momenta. The open (solid) points represent the results before (after) the correction described in the text. b) The $3 K \pi$ invariant mass distribution for selected candidates. The line represents the result of the fit described in the text, with the dot-dashed (dotted) line showing the signal (background) component.

$\Delta m u^{q} e^{a u}$, with $u=\left(\Delta m / m_{\pi}\right)-1$, to account for $\pi_{s}^{+}$decays in flight. The parameters $a, q$ and those of the Gaussian functions are fixed from simulation, except for an overall scale factor applied to the Gaussian widths. The background function is $B(\Delta m)=\Delta m \sqrt{u} e^{c u}$, with $C$ the only free parameter.

The $\Delta m$ distributions are shown along with the fit results in Fig. 3. Both fits have good quality and return signals of $138,539 \pm 109$ and $174,286 \pm 50$ decays, respectively, with width scale factors of $1.06 \pm 0.01$ and $1.08 \pm 0.01$. The $D^{*}(2010)^{+}$width is measured from the Breit-Wigner tails, rather than the central peak, of the $\Delta m$ distribution, which is made possible by the high signal:noise ratio. The two $D^{0}$ decay modes give consistent results, which we average.

\section{Results and conclusions}

We estimate systematic uncertainties due to energy loss as described above, and by varying the shapes and parameters of the signal and background functions. In addition, we use disjoint subsets in three variables chosen in advance to address specific issues of detector uniformity and resolution: the azimuthal angle $\phi$ of the $D^{0}$ or $D^{*}$ candidate momentum in the detector; the candidate momentum itself; and $\Delta m$ for the $m_{D^{0}}$ measurement or $m_{K \pi(\pi \pi)}$ for the others.

For the $D^{0}$ mass, our primary result is the $Q$ value

$$
Q=m_{D^{0}}-3 m_{K^{ \pm}}-m_{\pi^{ \pm}}=244.240 \pm 0.048 \text { (stat.) } \pm 0.041 \text { (syst.) } \mathrm{MeV} / c^{2},
$$

where the systematic uncertainty is dominated by the energy loss $\left(31 \mathrm{keV} / c^{2}\right)$ and $\Delta m$ variation $\left(28 \mathrm{keV} / c^{2}\right)$. The corresponding $m_{D^{0}}=1864.841 \pm 0.063$ (exp.) $\pm 0.046\left(m_{K^{ \pm}}\right)$has a large contri- 

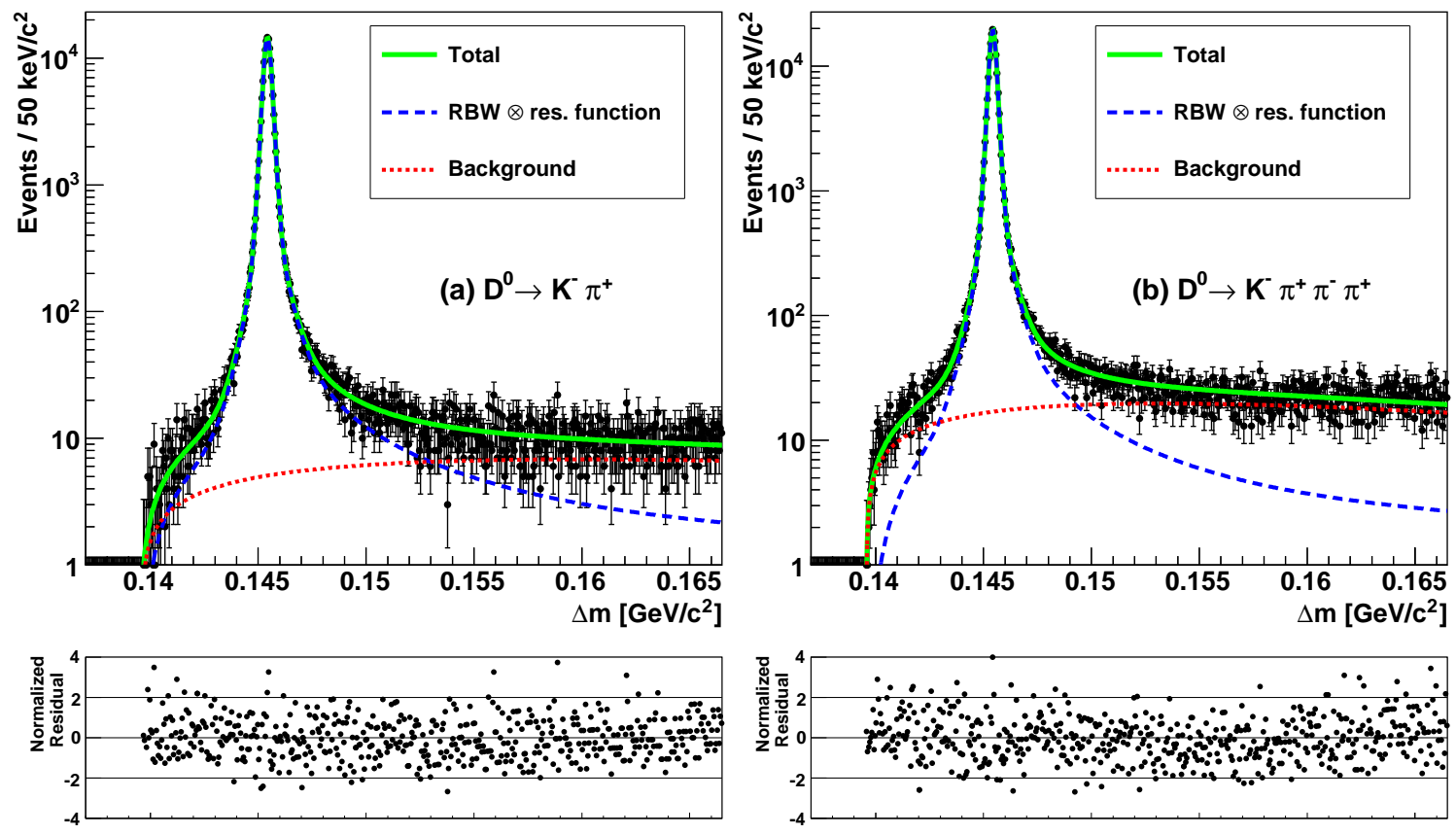

Figure 2: Distribution of the reconstructed mass difference for selected $D^{*+} \rightarrow D^{0} \pi_{s}^{+}$candidates in the (a) $D^{0} \rightarrow K^{-} \pi^{+}$and (b) $D^{0} \rightarrow K^{-} \pi^{+} \pi^{-} \pi^{+}$decay modes. The solid lines represent the results of the fits described in the text, with the dashed (dotted) lines showing the signal (background) components.

bution from current knowledge of the charged kaon mass [3]. Our result, with a total uncertainty of $78 \mathrm{keV} / c^{2}$ is consistent with, and twice as precise as, the current world average [3].

For the $D^{*}(2010)^{+}$natural width and the $D^{*}(2010)^{+}-D^{0}$ mass difference, we measure

$$
\Gamma_{D}^{*}=83.3 \pm 1.3 \text { (stat.) } \pm 1.4 \text { (syst.) } \mathrm{keV} \text { and } \Delta m=145425.8 \pm 0.5 \text { (stat.) } \pm 0.8 \text { (syst.) } \mathrm{keV} / c^{2} \text {. }
$$

The systematic uncertainty on $\Gamma$ is dominated by the $\phi$, momentum and $m_{K \pi(\pi \pi)}$ variations, while that on $\Delta m$ is dominated by energy loss and $\phi$ variation. Our $\Delta m$ result is consistent with the current world average [3], and a factor of five more precise. Our $\Gamma$ value is consistent with the only previous measurement, and a factor of twelve more precise. We can use this result, along with our previous measurements of the $D_{1}(2420)^{0}$ and $D_{2}^{*}(2460)^{0}$ widths to test the prediction of Ref. [4] that these mesons should respect a universal coupling $g=\Gamma / R$. Using $R$ values from [4] and our measured widths, we calculate $g_{2010}=0.76 \pm 0.01, g_{2420}=1.40 \pm 0.03$ and $g_{2460}=1.15 \pm 0.01$, which enlarges the discrepancy seen previously in the latter two states.

\section{References}

[1] J.P. Lees, et al. (BABAR Collaboration), arXiv:1304.5009 [hep-ex], submitted to Phys. Rev. D.

[2] B. Aubert et al. (BABAR Collaboration), Nucl. Instr. Methods A 479, 1 (2002); arXiv:1305.3560 [hep-ex], submitted to Nucl. Instr. Methods.

[3] J. Beringer, et al. (Particle Data Group), Phys. Rev. D 86, 010001 (2012).

[4] M. Di Pierro and E. Eichten, Phys. Rev. D 64, 114004 (2001). 